\title{
MedienPädagogik
}

Zeitschrift für Theorie und Praxis der Medienbildung

www.medienpaed.com

ISSN 1424-3636

Themenheft Nr. 37: Medienpädagogik als Schlüsseldisziplin in einer mediatisierten Welt. Perspektiven aus Theorie, Empirie und Praxis Herausgegeben von Henrike Friedrichs-Liesenkötter, Lara Gerhardts, Anna-Maria Kamin und Sonja Kröger

\section{Medienkritik zwischen Medienkompetenz und Media Literacy}

\author{
Medien- und subjektspezifische Einflüsse auf die \\ medienkritische Decodierungsfähigkeit
}

Sonja Ganguin, Johannes Gemkow und Rebekka Haubold

\section{Zusammenfassung}

Dorothee Meister folgerte auf der Basis einer theoretischen Diskussion über das Konzept der Medienkompetenz im vierten Jahrbuch zur Medienpädagogik, dass «die Herausforderung für die Pädagogik im Spannungsverhältnis zwischen Kompetenz und Performanz [besteht] und damit in der 〈Nichtidentität〉 eines universalen Regelsystems und regelgeleiteter aktueller Strukturierung in einer konkreten Sprechsituation» (Meister, Hagedorn, und Sander 2005, 171). Diese Schlussfolgerung ist kein triviales Zwischenfazit, sondern die Formulierung einer zentralen und zeitlosen Aufgabe der Medienpädagogik. Nach wie vor - und vermutlich auch mehr als je zuvor - steht die Medienpädagogik im Allgemeinen und das Konzept der Medienkompetenz im Speziellen vor der Aufgabe, einen tiefgreifenden Wandel von medientechnologischer Kommunikation pädagogisch zu begleiten. Ziel dieses Beitrages ist es, die inhärenten Strukturen der Konzepte Medienkompetenz und Media Literacy offenzulegen, um damit das «Spannungsverhältnis zwischen Kompetenz und Performanz» (ebd.) am Beispiel der medienkritischen Decodierungsfähigkeit zu lockern. Diese 〈Lockerung〉 folgt der Idee einer medien- und subjektspezifischen Ausdifferenzierung von medienkritischer Decodierungsfähigkeit mit Fokus auf digitalen Medien und medialen 
Handlungsräumen. Dazu werden zum einen die drei theoretischen Konzepte Kompetenz-Performanz, Medienkompetenz und Media Literacy vorgestellt. Zum anderen wird am Gegenstand von Medienkritik die Bedeutung von medien- und subjektspezifischen Einflüssen auf die Dimension der medienkritischen Decodierungsfähigkeit diskutiert.

\title{
Media Critique between Media Competence and Media Literacy. The Impact of Media and Subject Specifics on the media-critical decoding
}

\begin{abstract}
Based on a theoretical discussion, Dorothee Meister concluded in the fourth yearbook of media pedagogy, that "the challenge of pedagogy is the tension between competence and performance, thus in the «non-identity) of universal rules and the actual rule-based structuring of concrete speech» (Meister, Hagedorn, and Sander 2005, 171). This is rather a major and timeless challenge for media pedagogy than a preliminary conclusion. Still, and probably more than ever, media pedagogy in general and media competence in particular, face the task to address a deepening change of media-technological based communication. The goal of this contribution is to loosen «the tension between competence and performance» (ibid.) using the example of media-critical decoding. This 〈loosening〉 follows the idea of a differentiation of media-critical decoding by media, subject and situational specifics concerning digital media. First, the three theoretical concepts competence-performance, media competence and media literacy and their immanent structures will be presented. Second, by means of media critique the impact of media and subject specifics on media-critical decoding will be discussed.
\end{abstract}

\section{Zum Spannungsverhältnis von Kompetenz und Performanz}

Der Kompetenzbegriff hat seinen Ursprung in der Biologie und bezeichnet dort die Fähigkeit und die «Bereitschaft embryonaler Zellen, auf einen bestimmten Entwicklungsreiz zu reagieren»(Baacke 1975, 261). Die 
Übernahme des Kompetenzbegriffes aus der Biologie in den sozialwissenschaftlichen Diskurs geht auf den US-amerikanischen Linguisten Chomsky (1957) zurück. Dessen Kompetenz-Performanz-Modell ist in der PsychoLinguistik verortet. Chomsky formulierte die These, dass der Mensch über eine grundlegende Sprachkompetenz verfüge und geht dabei von der Annahme angeborener universaler Strukturen eines ¿Spracherwerbsapparates` aus. Für ihn ist die Sprachkompetenz die genetisch bedingte Fähigkeit die Sprache zu erlernen. Kompetenz beziehe sich auf kognitive Elemente, über die jeder Mensch verfüge und meine die Gesamtheit der Kenntnisse (auch der nicht bewussten), die eine sprechende/hörende Person von der eigenen Sprache hat. Er versteht unter Kompetenz in Anlehnung an Descartes und Humboldt die Fähigkeit von Sprechenden, nach bestimmten Regeln Sätze verschiedener Art zu bilden. Diese müssten sinnvoll aufgebaut sein, um von anderen verstanden zu werden (Baacke 1996). Anders als es die damals gängigen lernpsychologischen Modelle implizieren (beispielsweise das Reiz-Reaktions-Modell des Behaviorismus), sind Menschen so potenziell in der Lage, beliebig viele (unterschiedliche) Sätze zu generieren. Chomsky sieht Sprache als ein Mittel, um alle Gedanken zum Ausdruck zu bringen und auf jede neue Situation adäquat sprachlich zu reagieren. Dabei geht er davon aus, dass jeder Mensch über das Mittel einer (Universalsprache〉 verfügt. Kompetenz ist in Chomskys Modell das sprachliche Wissen. Sie steht in einem Wechselverhältnis, einer Spannung zu der Verwendung dieses Vermögens, was als Performanz oder sprachliches Können bezeichnet wird. Performanz meint den aktuellen Gebrauch, den ein Sprecher in einer konkreten Situation von seiner Kompetenz macht. Somit stellt die Performanz im Unterschied zur Kompetenz, die Leistung dar, Sätze zu verstehen und auch zu bilden. Dementsprechend ist Performanz die tatsächlich erbrachte Leistung, der Output eines Systems im Verhältnis zum Input, zu den Zielvorgaben oder dem von der Systemstruktur her Möglichen. Somit dient das sprachwissenschaftliche Kompetenz-Performanz-Modell von Noam Chomsky allgemein zur Unterscheidung von Kompetenz und Performanz, also dem Wissen um Regeln und deren konkrete Anwendung in einem aktuellen Sprechereignis.

Kritische Stellungnahmen zu Chomskys Ansatz laufen hauptsächlich darauf hinaus, sein Kompetenzbegriff bleibe zu «abstrakt, unhistorisch 
und apersonal» (Kübler 1996, 128); denn empirisch erforschen lassen sich nur die erzeugten sprachlichen Äusserungen. Hieraus ergibt sich das Spannungsfeld zwischen jenen spezifischen Performanzen im Gegensatz zu meist universell angesetzten Kompetenzen.

\section{Medienkompetenz zwischen Subjektideal und Subjektspezifik}

Das deutsche Medienkompetenzverständnis baut insbesondere auf den Arbeiten von Dieter Baacke (1975; 1996) auf. Dieser versteht Medienkompetenz als Teil der kommunikativen Kompetenz (Habermas 1971). Die kommunikative Kompetenz nach Baacke bezieht sich dabei nicht nur auf das Sprachverhalten, «sondern auch [auf] andere Arten des Verhaltens (z.B. Gesten, Expressionen durch leibgebundene Gebärde, auch Handeln)» (Baacke 1975, 261). Für Baacke gilt der Mensch als 〈homo educandus〉, also als erziehungsfähiges, aber auch erziehungsbedürftiges Lebewesen (Baacke 1975, 363). In diesem Sinne tritt die Kompetenz als normatives Konzept auf, da es den Menschen pädagogisch begleiten will und als idealistisch, da es dem Menschen Kompetenz unterstellt und diese somit zu einer grundlegenden anthropologischen Eigenschaft macht (Baacke 1994, 240).

Im Mittelpunkt steht bei Baacke das handlungsfähige Subjekt. Kompetenz wird gleichermassen vorausgesetzt und als erstrebenswertes Ideal eingeschätzt. Die individuelle Ausprägung der kommunikativen Kompetenz bleibt dabei aber «ein Spielraum des Menschen für die Bildung unendlich vieler Sätze wie für potentiell unbegrenztes Kommunikationsverhalten» (Baacke 1975, 101-102). Demnach entwickelt ein Individuum seine universelle Fähigkeit unter dem Einfluss sehr unterschiedlicher Variablen subjektspezifisch. Allerdings stellt sich in diesem Zusammenhang die Frage, inwieweit Medienkompetenz zur genetischen Grundausstattung des Menschen gehört. Argumentiert man z.B. mit der Wissensklufthypothese, dann liegt die Vermutung nahe, dass Medienkompetenz kein angeborenes Muster darstellt, sondern der Habitus im Umgang mit Medien von Bildung und somit von Erziehung abhängig ist. 
Insgesamt geht Baacke davon aus, dass jeder Mensch als prinzipiell 〈mündiger Rezipient〉 zu betrachten sei, der zugleich als kommunikativkompetentes Lebewesen auch aktiv Medien nutze. In diesem Sinne meint Medienkompetenz nach Baacke «also grundlegend nichts anderes als die Fähigkeit, in die Welt aktiv aneignender Weise auch alle Arten von Medien für das Kommunikations- und Handlungsrepertoire von Menschen einzusetzen» (Baacke 1996, 119). Baacke betont weiterhin, dass kommunikative Kompetenz die Fähigkeit ausdrücke, «variable Verhaltensschemata zu produzieren» (Baacke 1975, 286). Damit akzentuiert er die Fähigkeit, prinzipiell situations- und medienübergreifend zu handeln. Somit legt er kommunikative Kompetenz als universell aus. Damit gehört für Baacke Medienkompetenz ebenfalls zur kommunikativen Kompetenz in dem Sinne, dass Medien eine Besonderheit kommunikativer Strukturen darstellen. Obwohl Baacke sein Konzept zur Medienkompetenz eng mit der kommunikativen Kompetenz verbunden sah, grenzte er es von diesem ab, indem er «die Veränderung der Kommunikationsstrukturen durch technisch industrielle Vorkehrungen und Erweiterungen» einbezog (Baacke 1996, 119), ohne diese jedoch konkret zu benennen.

In der Tradition der deutschen Medienkompetenz steht demnach ein idealisiertes Subjekt im Vordergrund, womit die Subjekte nicht individuellen, sondern universellen Massstäben unterliegen, die gleichwohl universell für jedes Medium gelten, lediglich ihre Performanzen seien zu unterscheiden. In Anlehnung an die klassischen Aufgabenfelder der Medienpädagogik hat Baacke (1996) eine Operationalisierung von Medienkompetenz vorgeschlagen, in der Medienkritik eine von vier Dimensionen darstellt.

\section{Media Literacy als Chance für eine Medienspezifik}

Media Literacy leitet sich vom englischen 〈literate〉 ab, also der Fähigkeit zum Lesen und Schreiben. Mit steigender Alphabetisierungsrate löste sich der herkömmliche Literacy-Begriff von seiner schriftgebundenen Bedeutung und wird heutzutage auf alle kommunikativen Zeichensysteme bezogen. In diesem Kontext verweist Media Literacy auf funktionelle Fähigkeiten (〈abilities〉) und Fertigkeiten (〈skills〉), alle medial vermittelten Kommunikationsformen zu nutzen, zu analysieren, zu evaluieren und $\mathrm{zu}$ produzieren (Aufderheide und Firestone 1993, 6). 
Im Gegensatz zum deutschen medienpädagogischen Kompetenzverständnis basiert die theoretische Grundlage von Literacy auf dem Konzept des US-amerikanischen Pragmatismus (Tulodziecki und Grafe 2012, 50). Dieser fokussiert Handlungen als zentralen Faktor für Problemlösungen. Der Fokus auf Handlungen ist auch dem deutschen Kompetenzverständnis nicht fremd. Im Konzept der Media Literacy werden Handlungen jedoch - im Unterschied zum deutschen medienpädagogischen Kompetenzverständnis - nach wie vor an Medienbotschaften geknüpft. Die Verknüpfung mit Medienbotschaften kann bis zurück auf die Pionierarbeit zur Media Literacy von Len Masterman verfolgt werden. Masterman hat in einem seiner massgeblichen Werke zu Media Literacy mit dem Titel Media Awareness Education: Eighteen Basic Principles (1989) Medien ins Zentrum seiner Betrachtung gerückt:

«The central unifying concept of Media Education is that of representation. The media mediate. They do not reflect but re-present the world. The media, that is, are symbolic sign systems that must be decoded. Without this principle, no media education is possible. From it, all else flows» (Masterman 1989).

Hieran wird der fundamentale Unterschied zur deutschen Medienkompetenz sichtbar. Der deutschsprachige Diskurs favorisiert, bedingt durch die theoretische Genese des Kompetenzbegriffes, ein subjektiv-idealistisches Kompetenzverständnis (Gapski 2001; Gemkow 2017), während für Media Literacy die Medienbedingtheit von Kommunikation zentral ist.

Dieses Verständnis von Media Literacy spiegelt sich auch in dessen unterschiedlichen Definitionen und Modellen wider. Viele der Media LiteracyModelle (z.B. Aspen Media Literacy Institute 2003; Potter 2004; MediaSmarts 2019) - aber auch Modelle zur Information Literacy (z.B. Eisenberg und Berkowitz 1990; SCONUL 2011; CILIP 2018) - bestehen aus der schrittweisen Decodierung medienspezifischer Codes. Dazu werden standardisierte Handlungsschritte formuliert. Eine der am häufigsten zitierten Definitionen von Media Literacy stammt vom Aspen Media Literacy Institute. Dieser Definition zufolge, ist Media Literacy «the ability to access, analyze, evaluate and create messages in variety of forms» (Livingstone 2004, 3). 
Ein weiterer Definitionsvorschlag zur Media Literacy stammt von W. James Potter (2004, 36-38). Er konzeptualisiert in seinem Modell sieben Fähigkeiten. Dazu gehören standardisierte Handlungsschritte wie

- «breaking down the message»,

- «judging the value»,

- «determining which elements are alike in some way»,

- «generalizing [a] pattern to all elements»,

- 《using general principles to explain particulars»,

- «assembling elements into a new structure», und

- «create a [...] accurate description capturing the essence of a message».

In diesem Verständnis von Media Literacy zeigt sich das Ziel, subjektübergreifende Handlungsschritte zu entwerfen, mit denen Medienbotschaften decodiert werden können. Anders als im deutschsprachigen Kompetenzverständnis rückt demnach neben dem Sender und dem Inhalt der jeweiligen Botschaft (〈message〉) das Medium mit seiner spezifischen Codierungsweise ins Zentrum der Analyse. Diese medienspezifische Orientierung von Media Literacy ist entsprechend auch für die medienspezifische Ausdifferenzierung von Medienkritik, als Teil von Medienkompetenz, zentral.

\section{Medienkritik als Gegenstand}

Um Medienkritik als Teil von Medienkompetenz zu diskutieren, werden im Folgenden zunächst Gemeinsamkeiten und Unterschiede von Medienkompetenz hinsichtlich der Dimension Kritik anhand der Ansätze von Baacke (1996), Tulodziecki (1997), Aufenanger (1997), Schorb (1997) und Moser (2010) referiert, die etwa zur gleichen Zeit und zwar Ende der 1990er Jahre entstanden sind. Den vorgestellten Ansätzen ist mit Ausnahme des Ansatzes von Tulodziecki gemein, dass sie hinsichtlich ihrer praktischen Umsetzung, bezogen auf die Vermittlungsebene von Medienkritik, abstrakt bleiben: Es werden keine direkten zu beobachtenden Handlungen (Performanz) benannt, die einer Operationalisierung von Medienkritik dienen könnten. Was sich demnach hinter dem Medienkritikbegriff im Sinne von Medienkompetenz genau verbirgt, wie sie sich performativ erkennen lässt und wie sie zu bewerten ist, bleibt vage. 
So ist den Ansätzen gemeinsam, dass zwar von Kritik, Beurteilung und Bewertung gesprochen wird, jedoch nicht, wie diese konkret auszusehen haben. Zwar fordert Moser (2010) beispielsweise, dass der Einzelne über Kriterien verfügen solle, um Medieninformationen auf ihre Stichhaltigkeit und Relevanz hin zu beurteilen, gibt aber keine Kriterien an die Hand, mit deren Hilfe eine solche Bewertung möglich wird. Er benennt bei den Kriterien ebenfalls nicht, ob sie sich je nach Medium unterscheiden, also medienspezifisch bzw. medienabhängig sind oder auf alle Medien gleich angewandt werden können. Ausserdem muss berücksichtigt werden, ob es Bedingungen gibt, die dazu beitragen, welche Bewertungskriterien einem Individuum überhaupt zur Verfügung stehen. Andernfalls wäre davon auszugehen, dass alle Menschen gleichsam über dieselben verfügen können, die Kriterien also nicht subjektspezifisch wären.

Nach Ganguin ist Medienkritik ein mehrdimensionales Konstrukt; unter Medienkritik verstehen wir das kritische Wahrnehmen (1), Decodieren (2), Analysieren (3), Reflektieren (4) und Beurteilen (5) von Medien, ihren Inhalten, Formaten, Genres und Entwicklungen (Ganguin 2006; 2004.; Ganguin und Sander 2018). Die zweite Dimension von Medienkritik, die Decodierungsfähigkeit, die in diesem Artikel im Mittelpunkt steht, bezieht sich auf die Entschlüsselung der Mediensprache (Codes, Symbole, Informationsarten, Metaphern, Muster) durch Symbolverständnis (sowie Sprachverständnis) und Gedächtnisleistung. Damit entspricht sie jenem kommunikativen Kompetenzverständnis, das sich in den genannten Performanzen ausdrückt und sich als konkretes Beispiel eignet, die Notwendigkeit einer Medien- und Subjektspezifik zu erörtern.

\section{Die Bedeutung der Medienspezifik für die Medienkritik}

Die Medienspezifik ist Ausdruck bestimmter historischer Medienarrangements, die «ganz unterschiedliche Inszenierungsformen verlangen und Nutzungsbedingungen beinhalten»(Krotz 2012, 43). Mit dem digitalen Wandel verändert sich nicht nur die medienvermittelte Kommunikation, sondern auch «die soziale Bedeutung von Zeit und Raum [...], die sozialen Beziehungen und Normen der Menschen, die Machtkonstellationen, Werte, Traditionen und sozialen Regeln» (Krotz 2008, 54). 
Damit ändern sich u.a. auch Medienbotschaften. Begünstigt wird dieser Prozess zum einen durch die Tatsache, dass Botschaften von Medien kommuniziert und repräsentiert werden. Zum anderen werden Medienbotschaften sozial konstruiert und stehen mithin in Abhängigkeit von sozialen Strukturen. Ändern sich soziale Strukturen, verändert sich auch die medienbasierte Kommunikation, da Kommunikation auf Normen, Machtkonstellationen, Werten und Traditionen beruht. Medienbotschaften stehen also im Wechselspiel - mit medienvermittelter Kommunikation einerseits und mit soziokulturellem Wandel andererseits.

In der empiriegeleiteten Konzeptualisierung ihres Medienkritikmodells hat Ganguin $(2004,3)$ ebenfalls auf die fehlende Medienspezifik in der deutschsprachigen Medienkompetenzdebatte hingewiesen:

«In der Studie ist darüber hinaus die These formuliert worden, dass die Medienspezifik die Fähigkeit zur Medienkritik beeinflussen könnte. [...] Dies bedeutet, dass man über medienspezifische Kriterien verfügen muss, nach denen die Bewertung eines Mediums vorgenommen werden kann.».

Medienkritische Decodierungsfähigkeit ist laut Ganguin (2004, 4) durch die «Decodierung der Mediensprache (Codes, Symbole, Informationsarten, Metaphern, Muster) durch Symbolverständnis (sowie Sprachverständnis) und Gedächtnisleistung» gekennzeichnet. Der Wandel der Mediensprache darf dabei nicht missverstanden werden als Umbruch zwischen zwei medienhistorischen Epochen. Vielmehr bezeichnet die Digitalisierung selbst einen Prozess, in dem immer wieder neue Formen medienspezifischer Sprache entstehen.

Die Digitalisierung zeichnet sich nicht nur durch die Codierung von diskreten Werten und diskreten Zeiten aus. Symbole, die über digitale $\mathrm{Me-}$ dien kommuniziert werden, beinhalten z.B. den variierenden textbasierten Netzjargon (z.B. Akronyme wie 〈afaik〉, 〈asap〉 oder 〈imho〉), die Etablierung von Piktogrammen in Form von Emojis oder die meist nur zeitweise Konventionalisierung zweckentfremdeter Bilder als Internet-Meme (z.B. Captain Picards 〈Facepalm〉, Kappas Gesicht als Kennzeichnung von Sarkasmus oder Chuck-Norris-Witze). Informationen unterliegen ebenso einem medienspezifischen Wandel, da sie durch Medien repräsentiert werden. 
Allein Informationen, die entweder als nicht greifbarer subjektiver Informationsvorrat vorstellbar sind oder aber - in Abhängigkeit von der Reichweite des gewählten Medienbegriffes - als oral vermittelte Informationen auftreten, sind nicht medial repräsentiert. Die Medienspezifik von Informationen lässt sich insbesondere an dem humangeschichtlich umfangreichsten Angebot an Informationen - der Wikipedia - aufzeigen. Auf der Wikipedia werden Informationen auf eine Weise generiert, dargestellt, prozessiert und gespeichert, die von den Spezifika der Digitalisierung und der Plattform selbst abhängen. Auf der Wikipedia gibt es ein nebeneinanderher von kollaborativen Formen der Generierung von Inhalten, welche mit Formen der meritokratischen Prozessierung gemischt werden. Gemäss dem Meritokratieprinzip haben bestimmte Gruppen von Nutzenden - auf Grundlage des zeitlichen Bestehens ihres Accounts und der Anzahl ihrer generierten Beiträge auf der Wikipedia - erweiterte Rechte und Eingriffsmöglichkeiten auf der Plattform.

Diese wenigen Beispiele zur veränderten Mediensprache geben einen Einblick, welche dynamischen Formen die medienkritische Decodierungsfähigkeit adressieren muss, um in einer - abstrakt formuliert - «konkreten Sprechsituation» (Meister, Hagedorn, und Sander 2005, 171) als pädagogische Bezugsgrösse Orientierung geben zu können. Dementsprechend erfordert jedes Verständnis von Medienkritik und - im erweiterten Blick auch Medienkompetenz - ein Verständnis der zugrundeliegenden Medienspezifik.

\section{Die Bedeutung der Subjektspezifik für die Medienkritik}

Ergänzend zu dem Fokus auf die Medienbotschaft gilt es, Medienkritik ebenso an die Subjekte und ihre Handlungsmöglichkeiten zu knüpfen. Die folgende Betrachtungsweise nimmt deshalb die Medienaneignung (Schorb und Theunert 2000) als Grundlage der Mensch-Medien-Interaktion. Im Prozess der aktiven Auseinandersetzung des Menschen mit dem Medium sind vonseiten der Subjekte gewisse Fähigkeiten und Fertigkeiten vorausgesetzt, um Medien jeglicher Art decodieren zu können. Für digitale Medien sind hierbei - zusätzlich zu den bisherigen - andere Herausforderungen zu beachten. Daraus ergeben sich neue Anforderungen an eine 
Medienkritikfähigkeit, die zu unterschiedlichen Zielgruppen (z.B. in ihrer didaktischen Ansprache aber auch normativen sowie persönlichen Massstäben) führt. Im Folgenden soll die Decodierungsfähigkeit in ihrer Abhängigkeit vom Subjekt - somit nicht subjektübergreifend, sondern subjektspezifisch - dargestellt werden.

Decodierungsfähigkeit lässt sich nicht entlang soziodemografischer Kenndaten bestimmen. Es ist nicht so, dass Frauen oder Männer, oder jene mit einem grösseren Einkommen ein besseres oder schlechteres Symbolverständnis an den Tag legen (van Deursen, van Dijk, und Peter 2011). Auch eine Korrelation mit dem Alter ist bereits widerlegt worden (ebd.; Youmans u.a. 2013). Um Unterschiede in der Decodierungsfähigkeit zu beschreiben, eignen sich am besten die Eigenschaften von Menschen, die direkt mit dem jeweiligen Medium der Aneignung verknüpft sind (Haubold und Ganguin 2017).

Ein Aspekt der Decodierungsfähigkeit umfasst die Gedächtnisleistung. Zielgruppen mit ähnlicher Gedächtnisleistung konnten bisher zur Genüge entlang der Entwicklungspsychologie eingeteilt werden. Mit der rasanten technischen Entwicklung der letzten Jahrzehnte scheinen diese Modelle ihre Erklärungskraft verloren zu haben. Kognitiv «fertig entwickelte» Erwachsene (Haubold 2019) stossen auf einmal an ihre Grenzen, da in neu aufkommender Technik nicht das Lang- sondern das Kurzzeitgedächtnis an Bedeutung gewinnt. Die schnelle Verfügbarkeit und nahezu Allgegenwart der Informationen entlastet das Langzeitgedächtnis, was für junge Menschen vorteilhaft ist, Erwachsene jedoch vor Herausforderungen stellt (Wieland und Renatus 2019). Diese Gedächtnisleistung ist unabhängig davon, ob jemand mit sozialen Medien aufgewachsen ist oder nicht, sondern referiert schlicht auf abnehmende kognitive Fähigkeiten ab dem Jugendalter.

Ein zweiter Aspekt der Decodierungsfähigkeit umfasst das Sprachverständnis. Es ist relevant, auf welchen medialen Wortumfang, welches Zeichenverständnis zurückgegriffen werden kann, welche Dialekte und Grammatiken dem Subjekt vertraut und bekannt sind. Ähnlich wie bei analogen Medien sind Unterscheidungskriterien hierbei beispielsweise der Kontakt oder die Vorerfahrungen, die jemand mit den jeweiligen Medien(inhalten) hatte. Mit der Teilhabe an sozialen Medien verändern 
sich jedoch das Sprachverständnis und die Decodierungsfähigkeit grundlegend. 〈Social media logic〉 bedeutet im Zusammenhang der Decodierung zum Beispiel, dass nicht allein die Sprache der Medien, sondern die Sprache derjenigen, die sie befüllen, gesprochen oder wenigstens verstanden werden muss. Es handelt sich dabei um kein neues Phänomen an sich, denn auch bei Buchherausgebern müssen Zwecke und Intentionen sowie Art und Weise reflektiert werden. Sind es jedoch statt einer Handvoll, zahlreiche Herausgeberinnen und Herausgeber, deren Habitus dynamischer ist, so folgen auch ihre Sprache und Kultur einer anderen Logik (Hepp, Berg, und Roitsch 2014). Während zum Beispiel die Bildzeitung zwar andere Ausdrücke in Form kürzerer Sätze verwendet, ist doch ihr Symbolsytem das gleiche wie in der FAZ oder der Süddeutschen. Im Social Media Bereich verändert sich dies fluider. Ikone wie Emoticons unterscheiden sich zwischen den Plattformen teilweise grundlegend in ihrer Bedeutung. Ein weiteres Beispiel sind Hashtags, die je nach Plattform unterschiedliche Funktionen annehmen und als Symbole oder Metaphern auftreten. Diese werden teils durch die Medienproduzierenden, hauptsächlich jedoch durch die Nutzenden und deren Verwendung in Wechselseitigkeit erschaffen, statt durch Redaktionsstatut und Auseinandersetzung zwischen Leitung und Redaktion. Ein Wissen um die Community ist ergo ein erheblicherer Faktor für die Decodierungsfähigkeit von Medienkritik als zuvor. Es bedarf nicht allein einer inhaltlichen, sondern auch einer technischen Übersetzung sowie einer Übersetzung, die sich der Medienkultur der jeweiligen Community widmet. So stellt es einen Unterschied dar, ob man Teil der Nutzendenkultur ist oder nicht, ob man sie kennt - oder zumindest «versteht».

Für Medienkritikfähigkeit bedeutet dies, nicht alle Subjekte als gleich zu betrachten - weder nach ihren Anforderungen noch nach ihrer Nutzung von Medienkritik, sondern gezielt Strategien zu entwickeln, die sich mit den Besonderheiten der Subjekte auseinandersetzen. Die Reflexion der eigenen Nutzung nimmt ihren Ausgang in den eigenen, individuellen Fähigkeiten und Fertigkeiten und lässt sich - besonders bei Erwachsenen schwierig universell bestimmen. 


\section{Fazit}

Der vorliegende Beitrag hat die Bedeutung von medien- und subjektspezifischen Aspekten für das Konzept der Medienkritik am Beispiel der Decodierungsfähigkeit diskutiert. Dabei wurde argumentiert, dass das Konzept der Medienkritik diese Aspekte nicht übergehen darf, um als medienpädagogische Zieldimension bestehen zu können.

Die Medienkritik kann dabei eine Position zwischen den Konzepten der Medienkompetenz und Media Literacy einnehmen. Dabei muss sie zugleich medienspezifisch aktuelle und sich dynamisch wandelnde Aspekte mit medienkritischen Kriterien adressieren, ohne dabei subjektspezifische Aspekte zu negieren oder diese mit althergebrachten soziodemographischen Normen zu idealisieren.

Mit der Erweiterung individueller Handlungsfähigkeit durch kommunikative Medientechnologien entstehen neue Handlungsräume, die gleichwohl Möglichkeiten offerieren als auch Anforderungen stellen. Diese Handlungsräume sind vorstrukturiert. Diese Vorstrukturierung ist spezifisch für jede Plattform bzw. jedes mediale Angebot und stellt Handlungsmöglichkeiten und -bedingungen. Handlungsmöglichkeiten werden beispielsweise durch datenökonomische Intentionen verschiedener Akteure gezielt gelenkt. Handlungsräume werden durch kapitalistische Marktmechanismen strukturiert. Medienkritik muss daher die jeweilige Medienspezifik reflektieren. Es gilt sich mit den jeweils gegebenen Medienumgebungen spezifisch auseinanderzusetzen, da Handlungsräume erst in konkreten Medienumgebungen auf ihre Strukturierung hin untersucht werden können. Dies schliesst für das Konzept der Medienkritik aber auch ein, Handlungsräume empirisch zu untersuchen, um ihnen gegebenenfalls ein adäquates medienpädagogisches Korrektiv gegenüberzustellen. Für die medienpädagogische Forschung bedeutet dies, dass die Modellierung und Operationalisierung von Medienkritik im Besonderen und Medienkompetenz im Allgemeinen auf medienspezifische Aspekte zurückgreifen muss. Das heisst, es müssen auch Medienkompetenzmodelle entwickelt werden, die sich an medienspezifischen sowie auch thematischen Besonderheiten orientieren. Mögliche Fragen lauten hierbei: Was bedeutet es entsprechend sich in sozialen Netzwerken kompetent zu bewegen oder auf digitalen Informationsplattformen? Die medienpädagogische Forschung steht hierbei vor der Aufgabe, sich in Zukunft stärker zu positionieren. 
Dies gilt ebenfalls für die Subjektspezifik. In der Auseinandersetzung mit konkreten Handlungsräumen entstehen Möglichkeiten sich zu positionieren. Erst im Wechselspiel zwischen den Handlungsräumen und der subjektiven Positionierung tritt das jeweilige Subjekt zutage, welches medienkritisch handeln muss. Damit geht ein Verständnis sich wandelnder Identitäten und Bedürfnisse einher, die sich je nach Medienumgebung unterscheiden können. Dies schliesst die Perspektive ein, Subjekte nicht als vorgegeben zu betrachten, sondern als sich (selbst) konstituierend. Somit bildet das hier vertretende Verständnis von Medienkritik eine Brücke zwischen Medienkompetenz und Media Literacy, indem es zwischen Subjektideal und Subjektspezifik als auch Medienuniversal und Medienspezifik vermitteln kann und somit das (Spannungsverhältnis zwischen Kompetenz und Performanz〉 zu lockern vermag.

\section{Literatur}

Aspen Media Literacy Institute. 2003. «Literacy for the 21st Century. An Overview \& Orientation Guide to Media Literacy Education». http://www.medialit.org/sites/default/files/mlk/o1_MLKorientation.pdf.

Aufderheide, Patricia, und Charles Firestone. 1993. Media literacy: A Report of the National Leadership Conference on Media Literacy. Washington, D.C: Communications and Society Program, the Aspen Institute.

Aufenanger, Stefan. 1997. «Medienpädagogik und Medienkompetenz - Eine Bestandsaufnahme». In Medienkompetenz im Informationszeitalter, herausgegeben von Enquete Kommission «Zukunft der Medien in Wirtschaft und Gesellschaft; Deutschlands Weg in die Informationsgesellschaft» Deutscher Bundestag, 4:15-22. Enquete-Kommission: Zukunft der Medien. Bonn.

Baacke, Dieter. 1975. Kommunikation und Kompetenz. Grundlegung einer Didaktik der Kommunikation und ihrer Medien. 2. Aufl. München: Juventa.

Baacke, Dieter. 1996. «Medienkompetenz - Begrifflichkeit und sozialer Wandel». In Medienkompetenz als Schlüsselbegriff, herausgegeben von Antje Von Rein, 11224. Bad Heilbrunn: Klinkhardt.

Chomsky, Noam. 1957. Syntactic Structures. The Hague: Mouton.

Deursen, Alexander J.A.M. van, Jan A. G. M. van Dijk, und Oscar Peter. 2011. «Rethinking Internet skills: The contribution of gender, age, education, Internet experience, and hours online to medium- and content-related Internet skills». Poetics 39 (2): 125-44.

Eisenberg, Michael B., und Robert E. Berkowitz. 1990. Information Problem-solving. The Big Six Skills Approach to Library \& Information Skills Instruction. Norwood, NJ: Ablex Pub. Corp. 
Ganguin, Sonja. 2006. «Vom Kritikbegriff zur Medienkritik». In Medienkritik heute. Grundlagen, Beispiele, Praxisfelder, herausgegeben von Horst Niesyto, Matthias Rath, und Hubert Sowa, 71-86. München: kopaed.

Ganguin, Sonja. 2004. «Medienkritik - Kernkompetenz unserer Mediengesellschaft». Ludwigsburger Beiträge zur Medienpädagogik 6. https://www.ph-ludwigsburg.de/fileadmin/subsites/ıb-mpxx-t-ol/user_files/Online-Magazin/Ausgabe6/Ganguin6.pdf.

Ganguin, Sonja, und Uwe Sander. 2018. «Medienkritik: Zur Genese eines reflexiven Umgangs mit Medien». In Medienkritik im digitalen Zeitalter, herausgegeben von Horst Niesyto und Heinz Moser, 139-50. München: kopaed.

Gapski, Harald. 2001. Medienkompetenz. Eine Bestandsaufnahme und Vorüberlegungen zu einem systemtheoretischen Rahmenkonzept. Wiesbaden: Westdeutscher Verlag.

Gemkow, Johannes. 2017. «Medienkompetenz und die Mediatisierung des Wissens. Zum Potenzial der Dispositivanalyse am Beispiel mediatisierter Wissensbestände». merzWissenschaft 6: 19-30.

Habermas, Jürgen. 1971. «Vorbereitende Bemerkungen zu einer Theorie der kommunikativen Kompetenz». In Theorie der Gesellschaft oder Sozialtechnologie. Was leistet die Systemforschung?, herausgegeben von Jürgen Habermas und Niklas Luhmann, 101-41. Frankfurt am Main: Suhrkamp.

Haubold, Rebekka. 2019. «Age-Independence for Media Pedagogy». Media Studies 10 (20): 89-106. https://hrcak.srce.hr/ojs/index.php/medijske-studije/article/ view/9596.

Haubold, Rebekka, und Sonja Ganguin. 2017. «The Elderly's Media Appropriation as Variable for Target Groups». Networking Knowledge: Journal of the MeCCSA Postgraduate Network 10 Nr. 1: 27-43.

Hepp, Andreas, Matthias Berg, und Cindy Roitsch. 2014. Mediatisierte Welten der Vergemeinschaftung, Kommunikative Vernetzung und das Gemeinschaftsleben junger Menschen. Wiesbaden: Springer VS.

Krotz, Friedrich. 2008. «Kultureller und gesellschaftlicher Wandel im Kontext des Wandels von Medien und Kommunikation». In Medienkultur und soziales Handeln, herausgegeben von Tanja Thomas, 43-62. Wiesbaden: VS.

Krotz, Friedrich. 2012. «Von der Entdeckung der Zentralperspektive zur Augmented Reality: Wie Mediatisierung funktioniert». In Mediatisierte Welten. Forschungsfelder und Beschreibungsansätze, herausgegeben von Friedrich Krotz und Andreas Hepp, 27-55. Wiesbaden: Springer VS.

Kübler, Hans-Dieter. 1996. «Kompetenz der Kompetenz der Kompetenz. Anmerkungen zur Lieblingsmetapher der Medienpädagogik». medienpraktisch 20 (2): 11-15.

Livingstone, Sonja. 2004. "Media Literacy and the Challenge of new Information and Communication Technologies». http://eprints.lse.ac.uk/1017/1/MEDIALITERACY.pdf.

Masterman, Len. 1989. «Media Awareness Education: Eighteen Basic Principles». https://www.medialit.org/reading-room/media-awareness-education-eighteen-basic-principles. 
MediaSmarts. 2019. «Digital Literacy Fundamentals. Canada's Centre for Digital and Media Literacy». http://mediasmarts.ca/digital-media-literacy/general-information/digital-media-literacy-fundamentals/digital-literacy-fundamentals.

Meister, Dorothee M., Jörg Hagedorn, und Uwe Sander. 2005. «Medienkompetenz als theoretisches Konzept und Gegenstand empirischer Forschung». In Jahrbuch Medienpädagogik 4, herausgegeben von Ben Bachmair und Claudia de Witt, 169-86. Wiesbaden: VS. https://doi.org/10.21240/mpaed/retro/2017.08.10.X.

Moser, Heinz. 2010. Einführung in die Medienpädagogik. Aufwachsen im Medienzeitalter. Wiesbaden: VS.

Potter, James W. 2004. «The Media Literacy Model». http://www.sagepub.com/sites/default/files/upm-binaries/4889_Potter_Chapter_3_Media_Literacy_Model.pdf.

Schorb, Bernd. 1997. «Vermittlung von Medienkompetenz als Aufgabe der Medienpädagogik». In Medienkompetenz im Informationszeitalter, herausgegeben von Enquete Kommission «Zukunft der Medien in Wirtschaft und Gesellschaft; Deutschlands Weg in die Informationsgesellschaft» Deutscher Bundestag, 4:63-75. Enquete-Kommission: Zukunft der Medien. Bonn.

Schorb, Bernd, und Helga Theunert. 2000. «Kontextuelles Verstehen der Medienaneignung». In Qualitative Kinder- und Jugendmedienforschung. Theorie und Methoden: ein Arbeitsbuch, herausgegeben von Bernd Schorb und Ingrid PausHasebrink, 33-57. München: kopaed.

SCONUL. 2011. «The SCONUL Seven Pillars of Information Literacy. Core Model for Higher Education». http://www.sconul.ac.uk/sites/default/files/documents/ coremodel.pdf.

Tulodziecki, Gerhard. 1997. Medien in Erziehung und Bildung. Grundlagen und Beispiele einer handlungs- und entwicklungsorientierten Medienpädagogik. Bad Heilbrunn: Klinkhardt.

Tulodziecki, Gerhard, und Silke Grafe. 2012. «Approaches to Learning with Media and Media Literacy Education - Trends and Current Situation in Germany». Journal of Media Literacy Education 4 (1): 44-60. https://digitalcommons.uri. edu/jmle/vol4/issı/5.

Wieland, Mareike, und Rebecca Renatus. 2019. «You don't have to be quick to be information literate: comparing cognitive search strategies between younger and older adults». In MedienPädagogik: Zeitschrift für Theorie und Praxis der Medienbildung 35 (Media literacy), 1-19. .https://doi.org/10.21240/mpaed/35/2019.10.16.X.

Youmans, Robert J., Brooek Bellows, Christian A. Gonzalez, Brittany Sarbone, und Ivonne J. Figueroa. 2013. «Designing for the Wisdom of Elders: Age Related Differences in Online Search Strategies». In Universal Access in Human-Computer Interaction. User and Context Diversity, herausgegeben von David Hutchison, Takeo Kanade, Josef Kittler, Jon M. Kleinberg, Friedemann Mattern, John C. Mitchell, und Moni Noar, 240-49. Berlin, Heidelberg: Springer. 